\title{
Deciphering The Molecular Mechanism of Long Non-Coding RNA HIFA-AS1 Regulating Pancreatic Cancer Cells
}

\section{He Zhang}

Training Center for Clinical Skills and Medical staff®General Hospital of Northern Theater Command Xinwei Wang

Training Center for Clinical Skills and Medical staff囚General Hospital of Northern Theater Command

\section{Yue Wu}

Training Center for Clinical Skills and Medical staffखGeneral Hospital of Northern Theater Command

Xuehua Li

Training Center for Clinical Skills and Medical staff囚General Hospital of Northern Theater Command

Rui Sun

Training Center for Clinical Skills and Medical staff囚General Hospital of Northern Theater Command Jing Tian ( $\nabla 13352459336 @ 163 . c o m$ )

Training Center for Clinical Skills and Medical staffखGeneral Hospital of Northern Theater Command

\section{Research Article}

Keywords: pancreatic cancer, long non coding RNA, HIFA AS1, overexpression, molecular mechanism

Posted Date: June 10th, 2021

DOI: https://doi.org/10.21203/rs.3.rs-533849/v1

License: () (1) This work is licensed under a Creative Commons Attribution 4.0 International License. Read Full License 


\section{Deciphering the molecular mechanism of long non-coding RNA HIFA-AS1 regulating pancreatic cancer cells}

He Zhang ${ }^{1,2}$, Xinwei Wang ${ }^{1}$, Yue $\mathrm{Wu}^{1}$, Xuehua $\mathrm{Li}^{1}$, Rui $\operatorname{Sun}^{1}$, Jing Tian ${ }^{1 *}$

${ }^{1}$ Training Center for Clinical Skills and Medical staff, General Hospital of Northern Theater Command, No.83, Wenhua Road, Shenhe District, Shenyang, 110016, China;

${ }^{2}$ Laboratory Animal Center, General Hospital of Northern Theater Command, No.83, Wenhua Road, Shenhe District, Shenyang, 110016, China.

* Corresponding author: Jing Tian; e-mail: 13352459336@163.com. 


\begin{abstract}
Background: HIFA-AS1, an antisense transcript of HIFla gene, is a 652-bp long noncoding RNA (lncRNA) which globally expressed in multiple tissues of animals. Recent evidence indicated that the HIFA-ASI was involved in tumorigenesis of several types of cancer, but there were no reports on pancreatic cancer (PC).

Results: In order to investigate whether the HIFA-AS1 could mediate the PC or not, it was overexpressed in a PC cell line (PANC-1), and a series of experiments including cell viability detection, flow cytometry, transwell migration, clone formation and wound healing were performed. Functionally, the results indicated that overexpression (OE) of HIFA-AS1 could inhibit proliferation and shift, and promote apoptosis of PC cells. Moreover, to explore underlying molecular mechanism of anti-tumorigenic actions of HIFA-AS1 in PC cells, the iTRAQ (isobaric tags for relative and absolute quantification) quantitative proteomics analysis was implemented and the results indicated that OE of HIFA-ASI globally affected the expression levels of multiple protein associated with metabolism of cancer. Moreover, the network analysis revealed that the most of these differentially expressed proteins (DEPs) were integrated, and severed essential roles in regulatory function.
\end{abstract}

Conclusions: In summary, HIFA-AS1 may exhibit a potential therapeutic effect on PC, and our study provided useful information in this filed.

Keywords: pancreatic cancer, long non-coding RNA, HIFA-AS1, overexpression, molecular mechanism 


\section{Introduction}

Pancreatic cancer (PC) remains one of the most common causes of cancer-related mortality [1] at the seventh in humans worldwide [2], with 5-year overall survival rate of less than 5\% [3]. In most cases, PC develops with usually clinically silent at the early stage, but the variable symptoms, local invasiveness, or metastases only develop at an advanced stage [4]. Nowadays, the therapeutic efficacy of PC treatment is still very limited, and far from satisfactory [5, 6]. Hence, in order to enhance the cure rate of PC, it is necessary to investigate the molecular mechanisms, which would provide new opportunities to improve effective therapeutic strategies against PC.

Long non-coding RNAs (lncRNAs), a kind of non-coding RNAs transcripts, comprises longer than $200 \mathrm{bp}$ without protein-coding potential [7-9]. Current studies have showed that IncRNAs could mediate gene expression via chromosome remodeling, transcription and post-transcriptional processes [10]. As so far, increasing evidences demonstrate that lncRNAs play an important role in regulating vital molecular mechanism [11] and biological functions of the cells [12, 13], such as proliferation, migration, invasion, cell cycle and apoptosis [14-16]. Without a doubt, various expression of lncRNAs could contribute to tumor development and progression [17], but its regulatory mechanism had not been completely investigated.

HIFA-ASl is an antisense transcript of HIFl $\alpha$ [18], and accumulating evidence has revealed that it plays a key role in proliferation and apoptosis of vascular smooth muscle cells [19-21], and human hepatic stellate cells [22]. Furthermore, it promotes tumor necrosis factor- $\alpha$-induced apoptosis [23], thereby affecting the occurrence and 
development of thoracic aortic aneurysm [24]. HIFIA-AS1 can regulate starvationinduced hepatocellular carcinoma cell apoptosis, promoting hepatocellular carcinoma (HCC) cell progression [25]. Therefore, HIFA-AS1 has the capacities to affect the occurrence and development of multiple types of cancer, but there is no report on the molecular regulation mechanism of HIFA-AS1 in PC.

In the current study, to explore whether HIFA-AS1 could regulate PC or not, we constructed overexpression (OE) plasmids containing HIFA-ASI, and transferred them to a PC cell line. Subsequently, a series of experiments, including cell viability detection, flow cytometry, transwell migration, clone formation and would healing were conducted. And the experimental results showed that OE of HIFA-ASI could inhibit proliferation and metastasis, and promote apoptosis of PC cells, comparing with the normal control (NC) cells. In order to further explore the molecular mechanism of HIFA-AS1 regulating PC cells, we collected cell samples from $\mathrm{OE}$ and $\mathrm{NC}$ groups for iTRAQ (isobaric tags for relative and absolute quantification) proteomics experiments. Here we report the results.

\section{Materials and methods}

\section{Cell culture}

The human pancreatic cancer cell line (PANC-1) was provided by Procell (Wuhan, China) and cultured in monolayers in Dulbecco's modified Eagle's medium (DMEM) (Invitrogen, Carlsbad, USA). All media were supplemented with $10 \%$ fetal bovine serum (Hyclone UT, USA) in the presence of $100 \mathrm{U} / \mathrm{ml}$ penicillin and $50 \mu \mathrm{g} / \mathrm{m}$ streptomycin (Beyotime, Shanghai, China) with humidified atmosphere of $5 \% \mathrm{CO}_{2}$ and $95 \%$ air at $37^{\circ} \mathrm{C}$. 


\section{Plasmid construction, Lentivirus package and transfection}

HIFA-ASI was cloned into the pcDNA3.1(+) vector using the restriction sites for KpnI (GGTACC) and XhoI (CTCGAG), and this 652 bp insert was verified by sequencing. Two $\mu \mathrm{g}$ of plasmids containing $H I F 1 A-A S 1$ were mixed with the lentivirus packaging plasmids pMDLg-pRRE, pMD2.G, and pRSV-Rev according to the previous standard protocol [26]. Subsequently, PANC-1 cells were infected with 20 multiplicity of infection (MOI) lentivirus for $24 \mathrm{~h}$ and incubated in fresh medium. The cells were washed with fresh complete media after $24 \mathrm{~h}$ and the efficiency OE of HIFIA-AS1 was verified by quantitative RT-PCR (qRT-PCR).

\section{RNA extraction and qRT-PCR}

Total RNA was extracted from the cells using TRIzol reagent (Ambion, Austin, USA) and further purified with two phenol-chloroform treatments, then treated with RQ1 DNase (Promega, Madison, USA) to digest DNA. The quality and quantity of the purified RNAs were determined using a Nano Photometer spectrometer with the absorbance at $260 \mathrm{~nm} / 280 \mathrm{~nm}$ and next were verified by $1.2 \%$ agarose gel electrophoresis. The cDNA was synthesized with random primers with the Highcapacity cDNA Reverse-Transcription Kit (Takara, Dalian, China), and real time PCR was implemented for detecting gene expressions using the designed primers in Table 1 with SYBR Green I dye (Qiagen, Hilden, Germany). The PCR conditions were as follows: pre-denaturation at $95^{\circ} \mathrm{C}$ for $1 \mathrm{~min}, 40$ cycles of denaturing at $95{ }^{\circ} \mathrm{C}$ for $15 \mathrm{~s}$, annealing at $60{ }^{\circ} \mathrm{C}$ for $30 \mathrm{~s}$ and elongation at $72{ }^{\circ} \mathrm{C}$ for $40 \mathrm{~s}$. The relative expression of genes was analyzed by the $2^{-\triangle \Delta \mathrm{CT}}$ method with the Actin as an internal control [27].

\section{Cell viability detection}

The viability of PANC-1 cell was evaluated using the CCK-8 assay (Solarbio, Peking, 
China) according to the instructions of the manufacturer. The cells were slightly seeded into the 96-well plates with $100 \mu \mathrm{l}$ suspension per well overnight. At 0, 24, 48 and 72 h, $10 \mu \mathrm{l}$ of CCK-8 solution was added to each well, and then the plates were incubated for $0.5 \mathrm{~h}$. At last, absorbance was measured at $450 \mathrm{~nm}$ by microplate reader (Bio-Rad, Hercules, USA).

\section{Flow cytometric detection}

The apoptosis of the PANC-1 cells were determined by flow cytometry with Annexin V-conjugated FITC Apoptosis detection kit (BD, Franklin Lakes, USA). After infection for $24 \mathrm{~h}$, cells were harvested and washed twice with PBS, then re-suspended in $5 \mu \mathrm{l}$ FITC-conjugated anti-Annexin $\mathrm{V}$ antibody and in $500 \mu$ l binding buffer with $5 \mu \mathrm{l}$ Propidium iodide (PI). Apoptosis was measured with a FACS calibur flow cytometer MoFLO XDP (Beckman, Hercules, USA).

\section{Transwell invasion}

For the transwell assay, the properties of migration of cells were evaluated by using 24well transwell plates (Corning, NY, USA). About $1 \times 10^{4}$ cells per well were seeded into the upper chamber with serum free medium in triplicate. Medium containing 10\% FBS $(300 \mu \mathrm{l})$ was added to the DMEM of $5 \% \mathrm{CO}_{2}$ and $95 \%$ air at $37^{\circ} \mathrm{C}$. After incubation for $24 \mathrm{~h}$, the medium were removed, and cells were fixed with $4 \%$ paraformaldehyde for $15 \mathrm{~min}$, stained with $0.1 \%$ crystal violet for $20 \mathrm{~min}$, and counted from five randomly chosen fields for each well by stereo microscope (Leica, Wetzlar, Germany).

\section{Wound Healing Assay}

After $12 \mathrm{~h}$ of the transfected cells were seeded in 6-well plates, confluent monolayers in each well were washed with PBS and created using a $200 \mu l$ sterile pipette tip to 
generate a wound. Wound healing was evaluated and photographed images were taken by 200 magnification a Zeiss microscope (Leica, Wetzlar, Germany) from each well at 0,24 , and $48 \mathrm{~h}$ post-injury time points after the wound was made.

\section{Cell clone formation assay}

PANC-1 cells were plated into 6-well plates ( 800 cells per well) and cultured for 10 14 days, then were digested at the logarithmic phase to make a single-cell suspension using culture medium. Cell were stained with $0.4 \%$ crystal violet (Bio Basic Inc., Markham, Canada). Finally, the number of colonies was calculated under an inverted microscope (Leica, Wetzlar, Germany).

\section{Western Blotting Analysis}

PANC-1 cells were collected and the homogenates were centrifuged for $30 \mathrm{~min}$ at $4{ }^{\circ} \mathrm{C}$, $12,000 \mathrm{rpm}$ with cell lysis buffer. Then the protein extracts were separated on $10 \%$ SDS-PAGE and transferred to polyvinylidene difluoride (PVDF) membranes. Membranes were incubated at room temperature for $1 \mathrm{~h}$ in a 5\% skim milk TBST blocking solution and incubated with agitation at $4{ }^{\circ} \mathrm{C}$ overnight with specific primary antibodies anti-GAPDH (Beyotime, Shanghai, China), anti-Bax (Cell signaling technology, Beverly, MA, USA ), anti-P53 ( Szybio, Wuhan, China), anti-Caspase 3

(Cell Signaling Technology), and anti-PARP-1 (Cell Signaling Technology). Next, membranes also were incubated with secondary antibodies conjugated by horseradish peroxidase (HRP) (Zhong san jinqiao, Beijing, China) for $50 \mathrm{~min}$ at room temperature. At last, protein bands were determined using the Western blotting detection system (GE Healthcare, Amersham, UK).

\section{iTRAQ quantitative proteomics analysis}


For protein extraction from the cells $\left(5 \times 10^{6}\right)$, the lysis buffer $(7 \mathrm{M}$ Urea/2M Thiourea/4\% SDS/40 mM Tris-HCl, $\mathrm{pH} 8.5$ ) that contains $1 \mathrm{mM}$ phenylmethanesulfonyl fluoride (PMSF) and $2 \mathrm{mM}$ ethylenediaminetetraacetic acid (final concentration) was added to the sample, mixed and incubated in ice for $5 \mathrm{~min}$. Subsequently, DL-Dithiothreitol (DTT) was added to a final concentration of $10 \mathrm{mM}$. The lysate was sonicated on ice for $20 \mathrm{~min}$, then centrifuged at $4{ }^{\circ} \mathrm{C}, 13,000 \mathrm{~g}$ for $25 \mathrm{~min}$. The supernatant was mixed with four volumes of precooled acetone and kept at $-20{ }^{\circ} \mathrm{C}$ overnight. After centrifugation, the protein pellets were air-dried and re-dissolved in $8 \mathrm{M}$ urea/100mM triethylamine borane $(\mathrm{TEAB})(\mathrm{pH}=8.0)$. Protein samples were reduced with $10 \mathrm{mM}$ DTT at $56{ }^{\circ} \mathrm{C}$ for $35 \mathrm{~min}$ and alkylated with $50 \mathrm{mM}$ iodoacetamide (IAM) in the dark at room temperature for $30 \mathrm{~min}$. The supernatant was transferred to a new centrifuge tube, and the protein precipitation was performed by acetone precipitation. The protein pellet was re-dissolved by adding $8 \mathrm{M}$ urea/100 mM TEAB ( $\mathrm{pH}=8.0)$ solution, and DTT was added to a final concentration for $10 \mathrm{mM}$, with reduction reaction at $56{ }^{\circ} \mathrm{C}$ for 25 min. Subsequently, IAM was added to a final concentration for $55 \mathrm{mM}$, and the alkylation reaction was carried out at room temperature for $40 \mathrm{~min}$ in the dark. The protein concentration was assessed by the Bradford method.

The $100 \mu \mathrm{g}$ protein were trypsin digested with trypsin each sample. After diluting the protein solution 5 times with $100 \mathrm{mM}$ TEAB, trypsin were added by a mass ratio of 1:50 (trypsin: protein) overnight at $37^{\circ} \mathrm{C}$. The peptides were desalted with $\mathrm{C}_{18}$ column after enzymolysis, and the desalted peptides were vacuum freeze-dried.

The mass spectrometry data was collected and analyzed using the Eksigent nanoLC 
system (SCIEX, USA) coupled to the TripleTOF 5600+ mass spectrometers. Samples were iTRAQ labeled as follows: NC-1, X1; NC-2, X2; NC-3, X3; OE-1, X4; OE2, X5; and OE-3, X6. And all of the labeled samples were mixed with equal amounts. Next, the polypeptide solution was added to analytical ChromXP $\mathrm{C}_{18}$ column (Bonna-Agela Technologies Inc., Wilmington, DE) $(5 \mu \mathrm{m}, 100 \AA$, 4.6×250 mm), and eluted at 300 $\mathrm{nl} / \mathrm{min}$ on a $\mathrm{C}_{18}$ analytical column $(3 \mu \mathrm{m}, 75 \mu \mathrm{m} \times 150 \mathrm{~mm})$ over 90 min gradient. The two mobile phases were used both buffer A (2\% acetonitrile / $0.1 \%$ formic acid / $98 \%$ $\left.\mathrm{H}_{2} \mathrm{O}\right)$ and buffer B (98\% acetonitrile / 0.1\% formic acid / $2 \% \mathrm{H}_{2} \mathrm{O}$ ). For Information Dependent Acquisition (IDA), the first-order mass spectrum was scanned with an ion accumulation for $250 \mathrm{~ms}$, at the same time the secondary mass spectrum of 30 product ion scans were collected with $50 \mathrm{~ms}$. The MS1 spectrum was acquired in the range 350$1500 \mathrm{~m} / \mathrm{z}$, and the MS2 spectrum was acquired in the range $100-1500 \mathrm{~m} / \mathrm{z}$. Precursor ions were set from reselection for $15 \mathrm{~s}$.

The original MS/MS file data were analyzed using ProteinPilot Software v4.5 (AB Sciex, Shanghai, China). For protein identification, the Paragon algorithm, which was integrated into ProteinPilot, was used against the UniProt/SwissProt database for database searching. The parameters were set as follows: the instrument was TripleTOF 5600+, iTRAQ quantification, and cysteine modified with IAM, and biological modifications were selected as ID focus, trypsin digestion, quantitate, bias correction, and background correction was used for protein quantification and normalization. For calculation of the false discovery rate (FDR), an automatic decoy database search strategy was used to estimate FDR using the proteomics system performance evaluation pipeline software (PSPEP, integrated into the ProteinPilot Software). Unique peptides were used for iTRAQ labeling quantification, and peptides with global FDR values from fit less than $1 \%$ were considered for further analysis. Within each iTRAQ run, 
differentially expressed proteins (DEPs) were determined based on the ratios of differently labeled proteins and $\mathrm{p}$ values provided by ProteinPilot, the $\mathrm{p}$ values were generated by ProteinPilot using the peptides used to quantify the respective protein. For the determination of DEPs, fold changes (FC) were calculated as the average comparison pairs among biological replicates. Proteins with FC larger than 1.2 and a $\mathrm{p}$ $<0.05$ were considered to be changes that are significantly different.

\section{Bioinformatics and annotations}

The biological and functional properties of all the identified proteins were analyzed by matching to NCBInr (http://www.ncbi.nlm.nih.gov/) and Swiss-Prot/UniProt (http://www.uniprot.org/) databases, and were mapped with Gene Ontology (GO, http://www.geneontology.org/) and the Cluster of Orthologous Groups of proteins (COGs, http://www.ncbi.nlm.nih.gov/COG/) databases. The pathway enrichment analysis and metabolic pathways about the identified proteins were annotated by Kyoto Encyclopedia of Genes and Genomes (KEGG) mapping (http://www.genome.jp/kegg/). STRING v10.1 (http://string-db.org/) was applied to explore and analyze the proteinprotein interaction (PPI) information of DEPs to evaluate the interactive associations. The PPI network was constructed and visualized using Cytoscape software (version 3.5.1; www.cytoscape.org).

\section{Statistical analyses}

All data analysis was performed using the SPSS16.0 statistical software as the mean \pm standard deviation. Statistically significant differences comparison between two groups means were analyzed by Student's t-test. $\mathrm{P}$ value $<0.05$ was considered to be statistically significant. 


\section{Data availability statement}

The datasets generated and/or analysed during the current study are available in the Pr otemXchange repository(Accession No: IPX0003153000). For the interview, the data sets could also be obtained from a web link:

https://www.iprox.org/page/PSV023.html;?url=1622727859237GpMj, with a code: E $5 \times \mathbf{T}$.

\section{Results}

\section{$H I F A-A S 1$ regulates the apoptosis and proliferation of $\mathrm{PC}$ cells}

To investigate the role of HIFA-AS1 in the PC cells, an OE vector containing the HIFAAS1 was transfected into PANC-1 cells. The qRT-PCR experiment was applied to measure the efficiency of $\mathrm{OE}$, and the results showed that expression levels of HIFAAS1 in OE cells was about 10000-fold more as compared with the normal control (NC) cells (Fig. 1A), demonstrating a successful establishment of human PC cells with OE of HIFA-ASI.

To explore whether the HIF-ASI regulate the proliferation of PC cells or not, the experiments including CCK-8, were conducted for PANC-1 cells from $\mathrm{OE}$ and $\mathrm{NC}$ groups. The results indicated that viability cells with HIF-AS1 OE was declined significantly, during varying time periods $(0,24,48$ and $72 \mathrm{~h})(\mathrm{P}<0.001)$ (Fig. 1B and C).

Furthermore, flow cytometry was used to determine whether HIF-ASI could affect apoptosis of PANC-1 cells or not. It (Fig. 1D and E) displayed that the OE of HIF1AAS1 significantly promoted apoptosis of PC cells, and the number of apoptotic cells 
obviously increased about 50\% compared with the NC group (Fig. 1D). The above results indicated that $H I F 1 A-A S 1$ had the capacities to regulate proliferation and apoptosis of the PC cells. Further, western blot analysis reported that the expression levels of Cleaved caspase-3, Bax, P53, and PARP-1A protein were higher in OE group (Fig. 1F), thereby inducing apoptosis in pancreatic cancer.

\section{$H I F A-A S 1$ regulates the migration of $\mathrm{PC}$ cells}

To further explore the role of HIF 1A-AS1 in regulating metastasis of PANC-1 cells, the transwell migration assays were performed. It showed that the migration ability of cells from OE group was reduced about 50\% ( $\mathrm{P}<0.001)($ Fig. 2A and $\mathbf{B})$. In terms of cell clone, the clones formed in $\mathrm{NC}$ had a greater cell number about $45 \%$ compared with OE (Fig. 2C and D). Cell migration was detected using Wound-healing assay in PANC-1 cells. The results from invasion assay showed that OE of HIF-AS1 promoted cell invasion after transfection for 24 and 48 hours (Fig. 2E). And the difference of the wound width after 24 hours of transfection is the most significant compared with the comparison, which exceeds the control by about $20 \%$ (Fig. 2F), suggesting a functional role for HIF1A-AS1 in inhibiting metastasis of the PC cells.

\section{The summary of iTRAQ proteomics analysis}

To explore the molecular mechanism of HIFA-AS1 mediating the proliferation, apoptosis and shift of PANC-1 cells, an iTRAQ was applied to uncover altered protein expressions and signaling pathways.

In total, the quality of the data obtained from the iTRAQ was analyzed using parameters 
such as coefficient of variation about repeatability, distribution of unique peptide, peptide length, and distribution of coverage (Table 2). First of all, for the repeatability, there is a little difference concentration of CV data between $\mathrm{NC}$ and $\mathrm{OE}$ groups, and the cumulative percentages of $\mathrm{CV}$ were $7.81 \%$ and $7.29 \%$ respectively, indicating that the PANC-1 samples in each group are more reproducible (Fig. 3A).

In accordance with unique peptide determined as the peptide identified only for one protein, the presence of the corresponding protein can be uniquely determined. Then for the distribution of unique peptide number, the two-coordinate distribution map showed the number of unique peptides contained in all the proteins identified in this assay. For example, when the $\mathrm{x}$-axis, left $\mathrm{y}$-axis and right $\mathrm{y}$-axis are 2, 646 and 26.25 respectively, it means that there are 646 proteins with 2 as the unique number of peptides, which account for $26.25 \%$ of the total number of proteins obtained (Fig. 3B). Subsequently, the length of the identified peptides was analyzed. The average length of the polypeptide was 11.56 and within a reasonable range. Moreover, the length of the identified peptides was mainly concentrated between 7 and 15, and 9 was the maximum number (Fig. 3C). In addition, the protein identification coverage could reflect the overall accuracy of the identification results indirectly. The different colored pie represented the percentage of proteins with different identification coverage ranges. It showed that $37.21 \%$ proteins were with the peptide coverage less than $10 \%$, and $39.51 \%$ proteins had more than or equal to $20 \%$ of the peptide coverage, with the average protein identification coverage being $19.53 \%$ (Fig. 3D). 
A total of 4872 proteins were identified in all samples, and 4738, 2475 and 2539 ones were annotated successfully by GO, COG and KEGG, respectively (Fig. 3E). Particularly, the GO enrichment for the 4738 annotated proteins was carried out, including cellular localization (CC) (Data not shown), molecular functions (MF) (Data not shown) and biological processes (BP). The BP classification showed that most of these proteins were enriched in cellular process (13.04\%), metabolic process (11.26\%), biological regulation $(8.55 \%)$, regulation of biological process $(8.13 \%)$, cellular component organization or biogenesis $(7.00 \%)$ and so on $($ Fig. 3F).

\section{Exploration of DEPs and functional analysis}

On basis of the relative quantitative results, 338 DEPs were found in OE VS NC according to $\mathrm{FC}$ and $\mathrm{p}$ value ( $\mathrm{FC} \geq 1.2$ or $\leq 0.83, \mathrm{p} \leq 0.05$ ), and the up-regulated and down-regulated ones were 183 (Table 3) and 155 (Table 4), respectively. The protein abundance distribution graph, and the volcano plot showed the proportion of DEPs in the total identified proteins (Fig. 4A and 4B). A hierarchical clustering analysis of DEPs was also performed (Fig. 4C).

In addition, KEGG enrichment for DEPs was implemented. It can be seen from the pie chart that the top 10 pathways were different among all up-regulated and downregulated proteins. KEGG pathway enrichment was also variable across the upregulated proteins group and down-regulated proteins group. The enriched pathways for up-regulated proteins, include "RNA transport" (ID: ko03013), "Metabolic pathways" (ID: ko01100), "Ribosome” (ID: ko03010), "Spliceosome” (ID: ko03040), "Microbial metabolism in diverse environments" (ID: ko01120), "Pathogenic 
Escherichia coli infection" (ko05130), "Protein processing in endoplasmic reticulum" (ko04141), “Purine metabolism” (ko00230), “Glycolysis/ Gluconeogenesis” (ko00010), "Focal adhesion" (ko04510) and so on.

Furthermore, down-regulated proteins were primary enriched in some pathways, including "Metabolic pathways" (ID: ko01100), "Protein processing in endoplasmic reticulum" (ID: ko04141), "Pathways in cancer" (ID: ko05200), “Arrhythmogenic right ventricular cardiomyopathy" (ID: ko05412), "Microbial metabolism in diverse environments" (ID: ko01120) and "Regulation of actin cytoskeleton" (ko04810), “Tight junction" (ko04530), "Hypertrophic cardiomyopathy (HCM)" (ko05410), "Peroxisome" (ko04146), "Dilated cardiomyopathy" (ko05414) and so on (Fig. 4D).

According to these results indicate that the inhibitory effects of HIFIA-AS1 on the proliferation, apoptosis and migration of PANC-1 cells may be related to its capability to regulate protein interactions, catalytic activity and enzyme regulator activity. The top 10 pathway metabolic function types were different in all up-regulated and downregulated differential proteins by KEGG. Four types were same containing metabolic pathway, regulation of actin cytoskeleton, microbial metabolism in diverse environment, protein processing in endoplasmic reticulum. Pathway analysis revealed that "Metabolic pathways" at the second of up-regulated genes and at the first in downregulated genes in the enrichment results. Moreover, OE of HIFIA-AS1 may exhibit anticancer effects by regulating the pathways associated with metabolism of cancer. Therefore these results suggested that $H I F 1 A-A S 1$ might affect RNA polymerase to 
control the transcription of downstream tumor-associated genes as to antagonize the proliferation, apoptosis and migration of PC cells.

\section{Construction of DEPs Protein-protein interaction (PPI) network}

PPI network of common DEPs was constructed by the STRING online database and Cytoscape software to analyze the interactions of DEPs because the String database could identify interactions between known proteins and predictive proteins (Fig. 5). A total of 338 DEPs (155 down-regulated and 183 up-regulated) were filtered into the DEPs PPI network complex. The wonderful network suggested that these DEPs might work together to regulate apoptosis, proliferation and invasion of PC cells. These proteins are expected to become targets for the treatment of PC.

\section{Expression Validation by qRT-PCR}

To confirm the veracity and reliability of the proteomic assays, the expression levels of five candidate proteins were measured by qRT-qPCR, including $M X 1$ (Interferoninduced GTP-binding protein Mx1), IFIH1 (Interferon-induced helicase C domaincontaining protein 1), IFIT1 ( Interferon-induced protein with tetratricopeptide repeats 1), ISG15 (Ubiquitin-like protein ISG15), P4HB (Protein disulfide-isomerase), SOD2 (Superoxide dismutase [Mn], mitochondrial) (Fig. 6). Some specific primers were designed for these candidate (Table 1). MXI, IFIH1, IFITI and ISG15 mRNAs showed more than 2-fold down-regulation as compared to NC (Fig 6A, B, C and E) and $P 4 H B$ more than 0.5- fold down-regulation (Fig. 6D). In addition, SOD2 showed 2 fold upregulation (Fig. 6F). In short, above data supported that the results of the proteome was 
of credibility.

\section{Discussion}

PC remains one of the deadliest cancer types and worlds' most aggressive malignancies [28]. Accumulating reports have reported that the potential of lncRNAs as diagnostic or prognostic biomarkers ubiquitously dysregulated and have crucial regulatory roles in tumor cells, including PC [29]. However, the regulatory mechanisms of multiple lncRNAs are elusive in many kinds of cancers such as thoracic aortic aneurysm and HCC. Herein, we first aimed to explore the molecular mechanisim of HIF1A-AS1 regulating PC.

In the present study, we investigated the biological function of HIFIA-AS1 on proliferation, apoptosis, and metastasis of PC cells. Consistently, it was found that HIF 1A-AS1 was a suppressor of cell growth and progression in PC. Firstly, upregulation of HIFIA-AS1 inhibited cell growth and promoted apoptosis in PANC-1 cancer cells. Moreover, HIFIA-AS1 inhibited cell migration. Actually, the function of HIF1A-AS1 in other tumors has been reported. For instance, higher expression of HIF 1A-AS1, a novel diagnostic predictor, could be clinically functioned as a potential biomarker in colorectal carcinoma [30]. Besides, the levels of HIFlA-AS1 were significantly increased in tumor tissues or serum from non-small cell lung cancer patients [31]. Above reports researched with clinical samples, but may be the opposite with this study using PC cells. Therefore, this study indicated HIFIA-ASI as an important role for a novel mechanism in the PC modulated progress, could develop as a potential therapeutic target or biomarker for PC prevention and control. 
The quantitative proteomics analysis revealed that the expression levels of SOD2 was up-regulated. Previous studies have revealed that SOD2 has both tumor suppressive and promoting functions, which are primarily related to its role as a mitochondrial superoxide scavenger and $\mathrm{H}_{2} \mathrm{O}_{2}$ regulator [32]. SOD2 is role as both a tumor suppressor in early tumorigenesis and as a tumor promoter during metastatic progression [33]. Therefore, HIF1A-AS1 could regulate SOD2 to inhibit the metabolic developmental process in PC. In addition, $M x l$ expression was inversely correlated with prostate cancer [34]. However, in this study the expression levels of $M X 1$ was positive correlation with PC. MX1 [35], the members of IFN-stimulated genes [36], HIF 1A-AS1 could regulate $M X 1$ of type I interferon-mediated signaling pathway to restrain PC. According to previous reports, IFIT1 [37], OE of IFIT1 involved in a variety of biological processes, such as cell proliferation, migration and tumor growth $[38,39]$. In this review, we hypothesize that HIF1A-AS1 could inhibit IFIT1 to regulate the cell proliferation, migration to restrain PC to some extents. IFIH1, may play an important role in enhancing natural killer cell function and may be involved in growth inhibition and apoptosis in several tumor cell lines [40, 41]. In a word, the involvement of IFIH1 in the growth inhibition and apoptosis of PANC-1 cancer cells was regulated by HIF1AAS1. ISG15, induce natural killer cell proliferation, act as a chemotactic factor for neutrophils and act as an IFN-gamma-inducing cytokine lays a substantial role in the antiviral state induced by IFN $[42,43]$. Further, we showed that inhibition of PC by HIF $1 A-A S 1$ and ISG15 is sufficiently. In line with these studies, the present findings demonstrated that HIF1A-AS1 might regulate some tumorigenesis of PANC-1 cancer 
cells via targeting interferon-mediated signaling pathway, ubiquitin system and $\mathrm{H}_{2} \mathrm{O}_{2}$ regulator all closely related to metabolic pathways in cancer. In addition, tumorsuppressive role HIFIA-AS1 positively regulated the expression of $S O D 2$, and negatively regulated the five gene expression related to metabolic regulation to suppress PC growth and progression.

In summary, these findings demonstrated that HIF IA-AS1 could inhibit cell growth and progression of PANC-1 cells. In the future studies about diagnostic specificity and sensitivity of HIFIA-AS1, may be used as a potential biomarker and guidance for early diagnosis of PC. Although clinical applications need to be further explored, these results further provided insight into the molecular mechanisms associated with the tumorigenesis and scientific experimental basis for the treatment of PC. 


\title{
Declarations
}

\section{Ethics approval and consent to participate}

Not applicable.

\section{Consent for publication}

Not applicable.

\begin{abstract}
Availability of data and materials
The datasets generated and/or analysed during the current study are available in the $\operatorname{Pr}$ otemXchange repository(Accession No: IPX0003153000). For the interview, the data sets could also be obtained from a web link:

https://www.iprox.org/page/PSV023.html;?url=1622727859237GpMj, with a code: $\mathbf{E}$ $5 \times \mathbf{T}$.
\end{abstract}

\section{Competing interests}

The authors declare that they have no competing interests.

\section{Funding}

Supported by Natural Science Funding of Liaoning Province (\#2019-ms-350) and SYDW [2018]09 Laboratory Animals Project Funding of Military.

\section{Author Contribution}

He Zhang, Rui Sun and Jing Tian designed and managed the project. He Zhang, Xinwei Wang and Yue Wu drafted the manuscript. Xuehua Li, Rui Sun and Jing Tian participated in sample collection and manuscript revision. All authors read and approved the final manuscript.

\section{Acknowledgement}


Supported by Natural Science Funding of Liaoning Province (\#2019-ms-350) and SYDW [2018]09 Laboratory Animals Project Funding of Military. 
Figure 1. Overexpression (OE) of $H I F A-A S 1$ affects the proliferation, apoptosis of the pancreatic cancer (PC) cells (Pan-1). (A) Real-time PCR showed that the levels of HIFIA-AS1 was significantly increased in the cells from OE group compared with normal control (NC). (B) \& (C) OE of HIFIA-AS1 reduces significantly the viability of PC cells at 24, 48, and $72 \mathrm{~h}$. (D) OE of HIF1A-AS1 promotes significantly apoptosis of PC cells assessment of cellular apoptosis using Annexin V - fluorescein isothiocyanate staining coupled with flow cytometry. (E) Total percentage of apoptotic PANC-1 cells in each group are summarized with data presented as the mean \pm SD of three independent experiments. (F) Western blotting reveal Cleaved caspase-3, Bax, P53, and PARP-1A protein expression in PANC-1 cells. $* * * \mathrm{P}<0.001$ 
Figure 2. Overexpression (OE) of $H I F 1 A-A S 1$ regulates metastasis of the pancreatic cancer (PC) cells (Pan-1). (A) \& (B) OE of HIF 1A-AS1 inhibits metastasis of PC cells by transwell migration assay. (C) \& (D) OE of HIFIA-ASI inhibits metastasis of PC cells by clone formation assays. (E) \& (F) OE of HIF1A-AS1 inhibits metastasis of PC cells by wound-healing assay. $(n=3$ cultures, paired Student's t-test, $\pm \mathrm{SD}) * * \mathrm{P}<0.01, * * * \mathrm{P}<0.001$ 
Figure 3. The summary of the iTRAQ-based proteomic analysis for HIF-AS1 overexpression (OE) and normal control (NC) groups. (A) A shows the comparison of the $\mathrm{CV}$ values of different experimental groups more intuitively through the $\mathrm{CV}$ box diagram. The box diagrams of different colors represent the CV distribution of different experimental groups. The median CV of each box from left to right is $7.81 \%$ and $7.29 \%$, respectively. (B) Distribution of the number of unique peptides obtained for all the proteins identified in this assay. (C) Peptide length distribution map of the identified peptides. (D) Sectors of different colors in the pie chart representing the percentage of protein with different ranges of identified coverage. (E) Statistical graph of different functions annotation results of. The abscissa indicates identification or different annotation methods, and the ordinate indicates the number of proteins corresponding to the abscissa. (F) GO analysis of the proportion of proteins involved in various biological processes. 
Figure 4. Exploration of differentially expressed proteins (DEPs) and functional analysis. (A) Analyzing distribution of quantifiable multiples about difference proteins, the abscissa represents the value of multiples of difference after logarithmic transformation with base 2 as the base. The expression level greater than 0 shows upregulated, and the expression level less than 0 shows down-regulated. (B) Volcano plot showing the distribution of proteins that are up-regulated (red) and down-regulated (blue) or showed no change (black) on overexpression of HIF-AS1. (C) Hierarchical clustering of proteins vs samples where rows represent the clustering of proteins, and columns represent the clustering of samples. As the protein abundance ratio changes from small to large, the heat map color shows a corresponding blue-white-red change. (D) KEGG analysis of the proportion of proteins involved in various biological processes. 
Figure 5. Protein-protein interaction (PPI) network based on the differentially expressed proteins (DEPs). The total of 338 DEPs were filtered into the DEPs PPI network complex using the STRING online database. The round nodes indicate individual proteins. 
Figure 6. The quantitative RT-PCR (qRT-PCR) validation of some differentially expressed proteins (DEPs) obtained from iTRAQ analysis in Overexpression (OE) of $\boldsymbol{H I F}$-ASI and normal control (NC) groups. The expression levels of the same DEGs including $M X 1$, IFIH1, IFIT1, P4HB, ISG15 and SOD2 were validated by RTqPCR and normalized against Actin expression level. Values are normalized to those from cells with control vector. $\mathrm{n}=3$, paired student's $\mathrm{t}$ test, $\pm \mathrm{SD} .{ }^{*} \mathrm{P}<0.1,{ }^{*} * \mathrm{P}<0.05$, ***P<0.001. MX1, Interferon-induced GTP-binding protein Mx1; IFIH1, Interferoninduced helicase $\mathrm{C}$ domain-containing protein 1; IFIT1, Interferon-induced protein with tetratricopeptide repeats 1 ; ISG15, Ubiquitin-like protein ISG15; $44 H B$, Protein disulfide-isomerase; SOD2, Superoxide dismutase [Mn], mitochondrial. 


\section{Tables and Table Legends}

Table 1. The genes and primers used for qRT-PCR experiments.

\begin{tabular}{lll}
\hline Gene & Forward primer (5'-3') & Reverse primer (5'-3') \\
\hline MX1 & GACAGGACCATCGGAATCTTGAC & GGGCTTCGGACAGGCTCAG \\
IFIH1 & GGTGGTGATGATGAGTATTGTGATG & AGATTATTCCTCGTGCTGATTCCTC \\
IFIT1 & GTGGACCCTGAAAACCCTGAATC & AGCGGACAGCCTGCCTTAG \\
T4HB & TGCTGGTGGAACTGAGCAACTTC & TCGGTGTGGTCGCTGTCG \\
ISG15 & GCACCACAGCAAGCACCAC & CCCGCTCACTTGCTGCTTC \\
SOD2 & GATATGACCACCACCATTGAACTTC \\
Actin & TGGACTTCGAGCAAGAGATG & GAAGGAAGGCTGGAAGAGTG \\
\hline
\end{tabular}

Table 2. The all original data from the iTRAQ was analyzed using parameters.

These data were uploaded in Table 2.

Table 3. The 183 up-regulated DEPs were analyzed in OE VS NC.

These data were uploaded in Table 3.

Table 4. The 155 down-regulated DEPs were analyzed in OE VS NC.

These data were uploaded in Table 4.

Supplementary. The all original data included Table 2, Table 3 and Table 4.

These data were uploaded in Supplementary. 


\section{References}

1. Kamisawa T, Wood LD, Itoi T, Takaori K: Pancreatic cancer. Lancet 2016, 388(10039):73-85.

2. Torre LA, Bray F, Siegel RL, Ferlay J, Lortet-Tieulent J, Jemal A: Global cancer statistics, 2012. CA Cancer J Clin 2015, 65(2):87-108.

3. Siegel RL, Miller KD, Jemal A: Cancer statistics, 2018. CA Cancer J Clin 2018, 68(1):7-30.

4. Ferlay J, Soerjomataram I, Dikshit R, Eser S, Mathers C, Rebelo M, Parkin DM, Forman D, Bray F: Cancer incidence and mortality worldwide: sources, methods and major patterns in GLOBOCAN 2012. Int J Cancer 2015, 136(5):E359-386.

5. Gzil A, Zarebska I, Bursiewicz W, Antosik P, Grzanka D, Szylberg L: Markers of pancreatic cancer stem cells and their clinical and therapeutic implications. Mol Biol Rep 2019, 46(6):6629-6645.

6. Xu R, Yang J, Ren B, Wang H, Yang G, Chen Y, You L, Zhao Y: Reprogramming of Amino Acid Metabolism in Pancreatic Cancer: Recent Advances and Therapeutic Strategies. Front Oncol 2020, 10:572722.

7. Huang X, Zhi X, Gao Y, Ta N, Jiang H, Zheng J: LncRNAs in pancreatic cancer. Oncotarget 2016, 7(35):57379-57390.

8. Duguang L, Jin $H$, Xiaowei $Q$, Peng $X$, Xiaodong W, Zhennan L, Jianjun $Q$, Jie $Y$ : The involvement of IncRNAs in the development and progression of pancreatic cancer. Cancer Biol Ther 2017, 18(12):927-936.

9. Luo L, Wang M, Li X, Tian J, Zhang K, Tan S, Luo C: Long non-coding RNA LOC285194 in cancer. Clin Chim Acta 2020, 502:1-8.

10. Guo W, Zhong K, Wei H, Nie C, Yuan Z: Long non-coding RNA SPRY4-IT1 promotes cell proliferation and invasion by regulation of Cdc20 in pancreatic cancer cells. PLoS One 2018, 13(2):e0193483.

11. Ding M, Fu Y, Guo F, Chen H, Fu X, Tan W, Zhang H: Long non-coding RNA MAFG-AS1 knockdown blocks malignant progression in breast cancer cells by inactivating JAK2/STAT3 signaling pathway via MAFG-AS1/miR-3196/TFAP2A axis. Int J Clin Exp Pathol 2020, 13(10):2455-2473.

12. Sanchez Calle A, Kawamura Y, Yamamoto Y, Takeshita F, Ochiya T: Emerging roles of long noncoding RNA in cancer. Cancer Sci 2018, 109(7):2093-2100.

13. Tang Q, Hann SS: HOTAIR: An Oncogenic Long Non-Coding RNA in Human Cancer. Cell Physiol Biochem 2018, 47(3):893-913.

14. Zhao X, Wang P, Liu J, Zheng J, Liu Y, Chen J, Xue Y: Gas5 Exerts Tumor-suppressive Functions in Human Glioma Cells by Targeting miR-222. Mol Ther 2015, 23(12):1899-1911.

15. Chi Y, Wang D, Wang J, Yu W, Yang J: Long Non-Coding RNA in the Pathogenesis of Cancers. Cells 2019, 8(9).

16. Qian CJ, Xu ZR, Chen LY, Wang YC, Yao J: LncRNA MAFG-AS1 Accelerates Cell Migration, Invasion and Aerobic Glycolysis of Esophageal Squamous Cell Carcinoma Cells via miR765/PDX1 Axis. Cancer Manag Res 2020, 12:6895-6908.

17. Yang G, Lu X, Yuan L: LncRNA: a link between RNA and cancer. Biochim Biophys Acta 2014, 1839(11):1097-1109.

18. Xu J, Zhang Y, Chu L, Chen W, Du Y, Gu J: Long non-coding RNA HIF1A-AS1 is upregulated in intracranial aneurysms and participates in the regulation of proliferation of vascular 
smooth muscle cells by upregulating TGF-beta1. Exp Ther Med 2019, 17(3):1797-1801.

19. He Q, Tan J, Yu B, Shi W, Liang K: Long noncoding RNA HIF1A-AS1A reduces apoptosis of vascular smooth muscle cells: implications for the pathogenesis of thoracoabdominal aorta aneurysm. Pharmazie 2015, 70(5):310-315.

20. Wang S, Zhang X, Yuan Y, Tan M, Zhang L, Xue X, Yan Y, Han L, Xu Z: BRG1 expression is increased in thoracic aortic aneurysms and regulates proliferation and apoptosis of vascular smooth muscle cells through the long non-coding RNA HIF1A-AS1 in vitro. Eur J Cardiothorac Surg 2015, 47(3):439-446.

21. Wang J, Chen L, Li H, Yang J, Gong Z, Wang B, Zhao X: Clopidogrel reduces apoptosis and promotes proliferation of human vascular endothelial cells induced by palmitic acid via suppression of the long non-coding RNA HIF1A-AS1 in vitro. Mol Cell Biochem 2015, 404(12):203-210.

22. Zhang QQ, Xu MY, Qu Y, Hu JJ, Li ZH, Zhang QD, Lu LG: TET3 mediates the activation of human hepatic stellate cells via modulating the expression of long non-coding RNA HIF1AAS1. Int J Clin Exp Pathol 2014, 7(11):7744-7751.

23. Wu Y, Ding J, Sun Q, Zhou K, Zhang W, Du Q, Xu T, Xu W: Long noncoding RNA hypoxiainducible factor 1 alpha-antisense RNA 1 promotes tumor necrosis factor-alpha-induced apoptosis through caspase 3 in Kupffer cells. Medicine (Baltimore) 2018, 97(4):e9483.

24. Zhang X, Li H, Guo X, Hu J, Li B: Long Noncoding RNA Hypoxia-Inducible Factor-1 AlphaAntisense RNA 1 Regulates Vascular Smooth Muscle Cells to Promote the Development of Thoracic Aortic Aneurysm by Modulating Apoptotic Protease-Activating Factor 1 and Targeting let-7g. J Surg Res 2020, 255:602-611.

25. Hong F, Gao Y, Li Y, Zheng L, Xu F, Li X: Inhibition of HIF1A-AS1 promoted starvation-induced hepatocellular carcinoma cell apoptosis by reducing HIF-1alpha/mTOR-mediated autophagy. World J Surg Oncol 2020, 18(1):113.

26. Cribbs AP, Kennedy A, Gregory B, Brennan FM: Simplified production and concentration of lentiviral vectors to achieve high transduction in primary human T cells. BMC Biotechnol 2013, 13:98.

27. Livak KJ, Schmittgen TD: Analysis of relative gene expression data using real-time quantitative PCR and the 2(-Delta Delta C(T)) Method. Methods 2001, 25(4):402-408.

28. Ilic M, Ilic I: Epidemiology of pancreatic cancer. World J Gastroenterol 2016, 22(44):96949705.

29. Zhou M, Ye Z, Gu Y, Tian B, Wu B, Li J: Genomic analysis of drug resistant pancreatic cancer cell line by combining long non-coding RNA and mRNA expression profling. Int J Clin Exp Pathol 2015, 8(1):38-52.

30. Gong W, Tian M, Qiu H, Yang Z: Elevated serum level of IncRNA-HIF1A-AS1 as a novel diagnostic predictor for worse prognosis in colorectal carcinoma. Cancer Biomark 2017, 20(4):417-424.

31. Tantai J, Hu D, Yang Y, Geng J: Combined identification of long non-coding RNA XIST and HIF1A-AS1 in serum as an effective screening for non-small cell lung cancer. Int J Clin Exp Pathol 2015, 8(7):7887-7895.

32. Kim YS, Gupta Vallur P, Phaeton R, Mythreye K, Hempel N: Insights into the Dichotomous Regulation of SOD2 in Cancer. Antioxidants (Basel) 2017, 6(4).

33. Miar A, Hevia D, Munoz-Cimadevilla H, Astudillo A, Velasco J, Sainz RM, Mayo JC: Manganese 
superoxide dismutase (SOD2/MnSOD)/catalase and SOD2/GPx1 ratios as biomarkers for tumor progression and metastasis in prostate, colon, and lung cancer. Free Radic Biol Med 2015, 85:45-55.

34. Brown SG, Knowell AE, Hunt A, Patel D, Bhosle S, Chaudhary J: Interferon inducible antiviral MxA is inversely associated with prostate cancer and regulates cell cycle, invasion and Docetaxel induced apoptosis. Prostate 2015, 75(3):266-279.

35. Andersson I, Bladh L, Mousavi-Jazi M, Magnusson KE, Lundkvist A, Haller O, Mirazimi A: Human MxA protein inhibits the replication of Crimean-Congo hemorrhagic fever virus. $J$ Virol 2004, 78(8):4323-4329.

36. Imaizumi T, Satake U, Miyashita R, Kawaguchi S, Matsumiya T, Seya K, Ding J, Tanaka H: Interferon-induced transmembrane protein 1 and Myxovirus resistance protein 1 are induced by polyinosinic-polycytidylic acid in cultured hCMEC/D3 human cerebral microvascular endothelial cells. J Neuroimmunol 2019, 337:577047.

37. Abbas YM, Pichlmair A, Gorna MW, Superti-Furga G, Nagar B: Structural basis for viral 5'PPP-RNA recognition by human IFIT proteins. Nature 2013, 494(7435):60-64.

38. Pidugu VK, Wu MM, Yen AH, Pidugu HB, Chang KW, Liu CJ, Lee TC: IFIT1 and IFIT3 promote oral squamous cell carcinoma metastasis and contribute to the anti-tumor effect of gefitinib via enhancing p-EGFR recycling. Oncogene 2019, 38(17):3232-3247.

39. Pidugu VK, Pidugu HB, Wu MM, Liu CJ, Lee TC: Emerging Functions of Human IFIT Proteins in Cancer. Front Mol Biosci 2019, 6:148.

40. Jiang M, Osterlund P, Sarin LP, Poranen MM, Bamford DH, Guo D, Julkunen I: Innate immune responses in human monocyte-derived dendritic cells are highly dependent on the size and the 5' phosphorylation of RNA molecules. J Immunol 2011, 187(4):1713-1721.

41. Zhang J, Liu X, Meng Y, Wu H, Wu Y, Yang B, Wang L: Autoimmune disease associated IFIH1 single nucleotide polymorphism related with IL-18 serum levels in Chinese systemic lupus erythematosus patients. Sci Rep 2018, 8(1):9442.

42. Zhao C, Denison C, Huibregtse JM, Gygi S, Krug RM: Human ISG15 conjugation targets both IFN-induced and constitutively expressed proteins functioning in diverse cellular pathways. Proc Natl Acad Sci U S A 2005, 102(29):10200-10205.

43. Hsiang $T Y$, Zhao $C$, Krug RM: Interferon-induced ISG15 conjugation inhibits influenza A virus gene expression and replication in human cells. J Virol 2009, 83(12):5971-5977. 
A

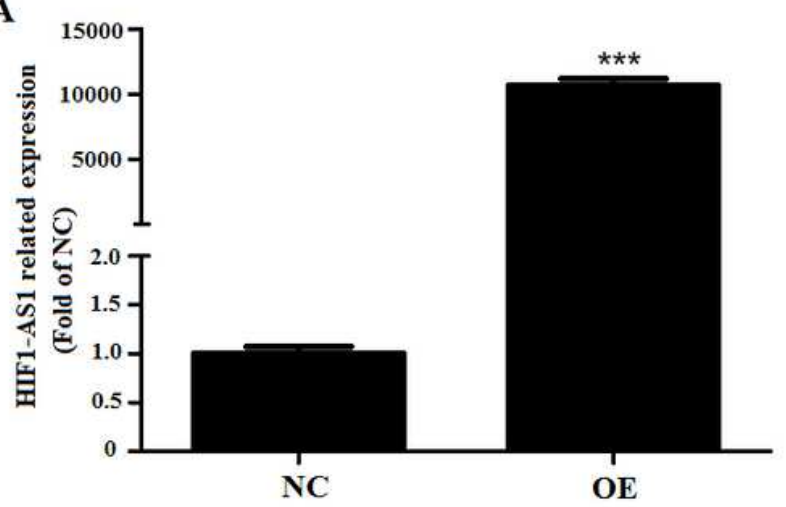

C

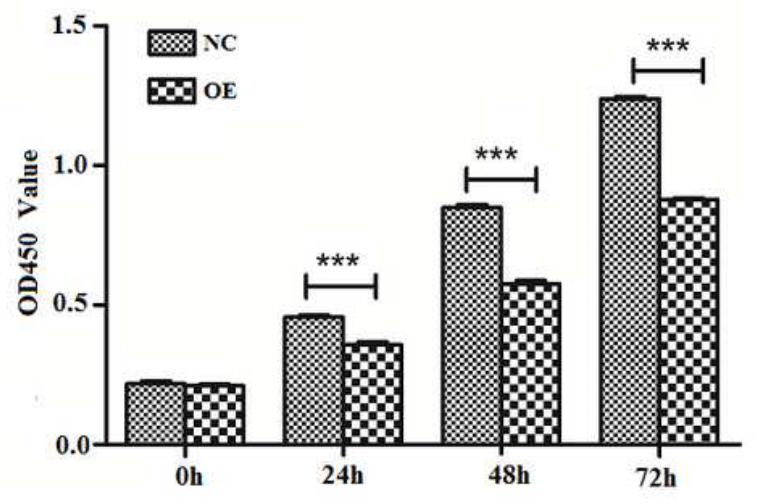

E

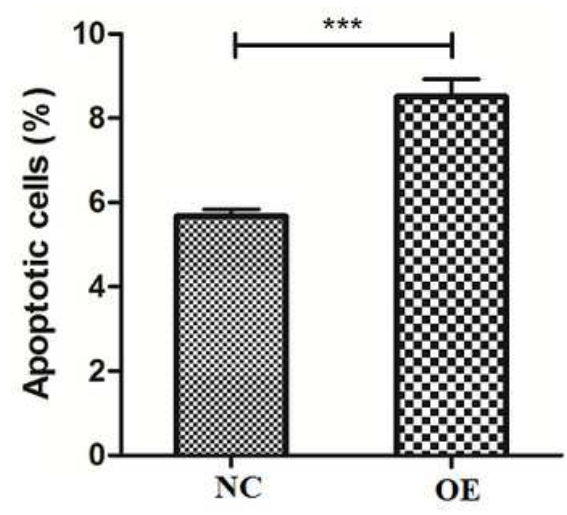

B

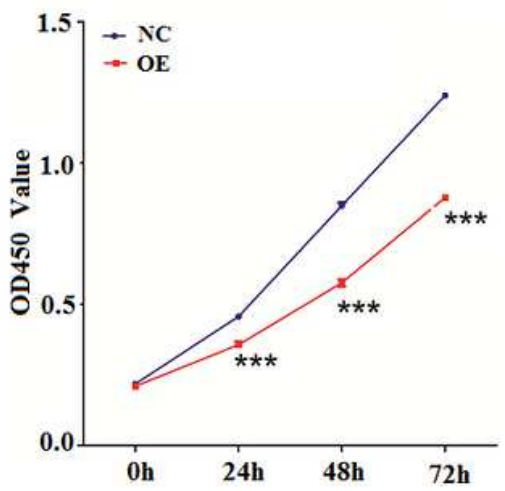

D

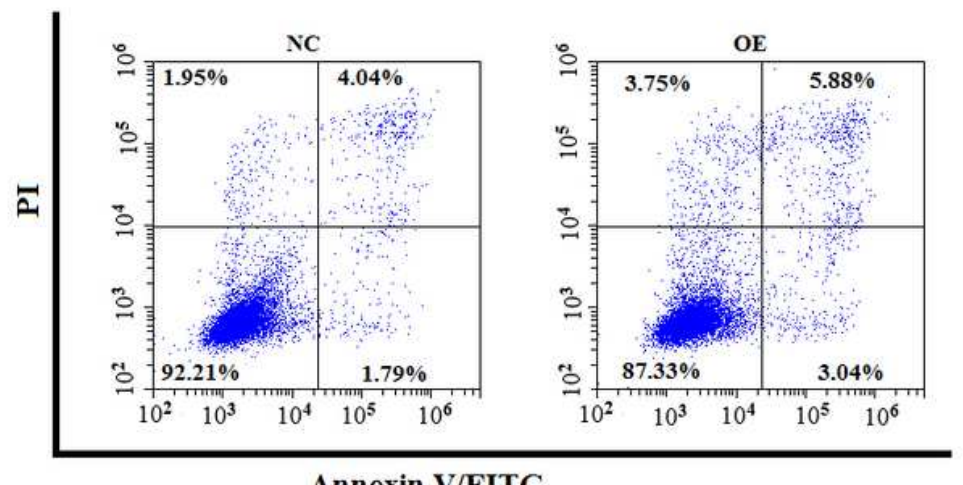

Annexin V/FITC

F

Figure 1

Overexpression (OE) of HIFA-AS1 affects the proliferation, apoptosis of the pancreatic cancer (PC) cells (Pan-1). (A) Real time PCR showed that the levels of HIF1A AS1 was significantly incre a sed in the cells from OE group compared with normal control (NC) NC). (B) \& C) OE of HIF1A AS1 reduces significantly the viability of PC cells at 24, 48, and $72 \mathrm{~h}$. (D) OE of HIF1A AS1 promotes significantly apoptosis of PC cells assessment of cellular apoptosis using Annexin $\mathrm{V} \otimes$ fluorescein isothiocyanate staining coupled with flow cytometry. ( Total percentage of apoptotic PANC 1 cells in each group are summarized with data 
presented as the mean \pm SD of three independent experiments. $(F)$ Western blotting reveal Cleaved caspase-3, Bax, P53, and PARP-1A protein expression in PANC 1 cells. ${ }^{* * *} \mathrm{P}<0.001$

A
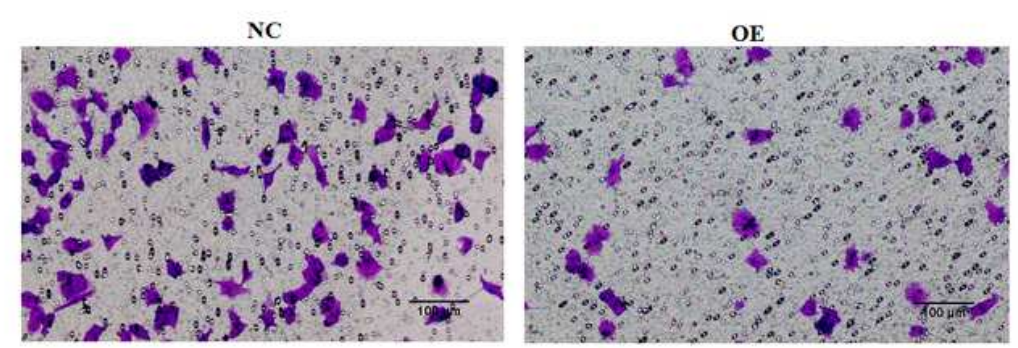

C

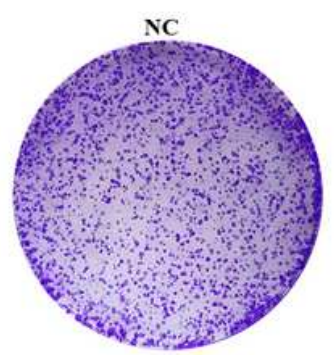

$\mathbf{E}$
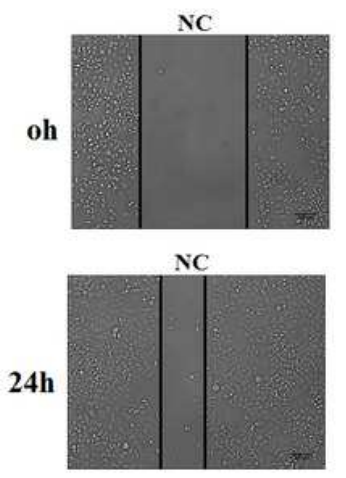

NC

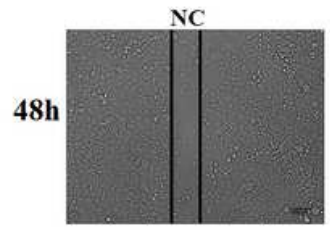

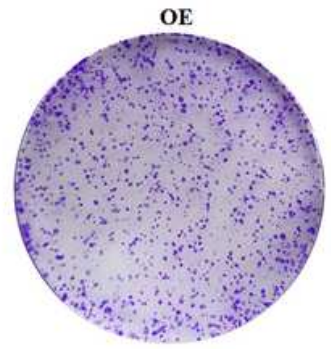

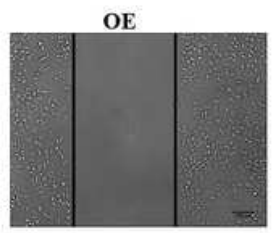

OE

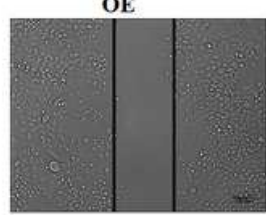

$\mathrm{OE}$

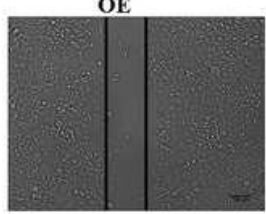

B

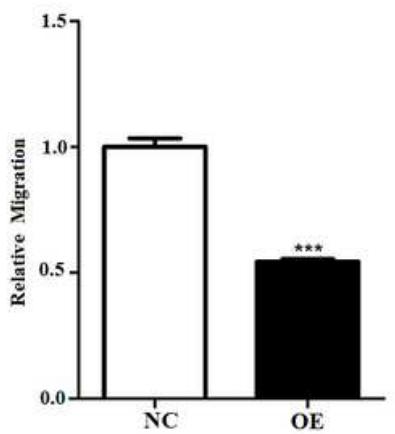

D

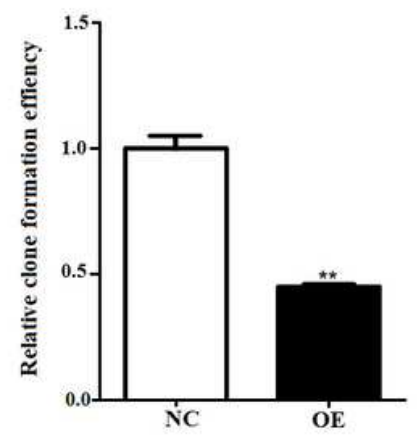

$\mathbf{F}$

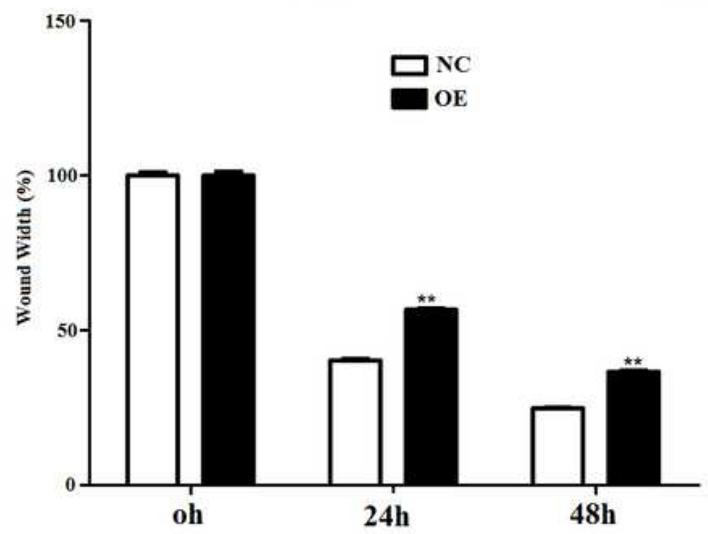

Figure 2

Overexpression (OE) of HIF1A-AS1 regulates metastasis of the pancreatic cancer (PC) cells (Pan-1). (A) \& (B) OE of HIF1A AS1 inhibits metastasis of PC cells by transwell migration assay. (C) \& (D) OE of HIF1A AS1 inhibits metastasis of PC cells by clone formation assays. (E) \& (F) OE of HIF1A AS1 inhibits

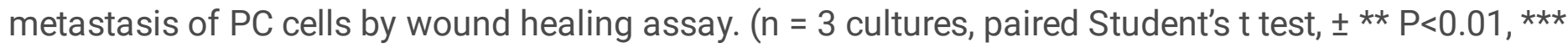
$\mathrm{P}<0.001$ 


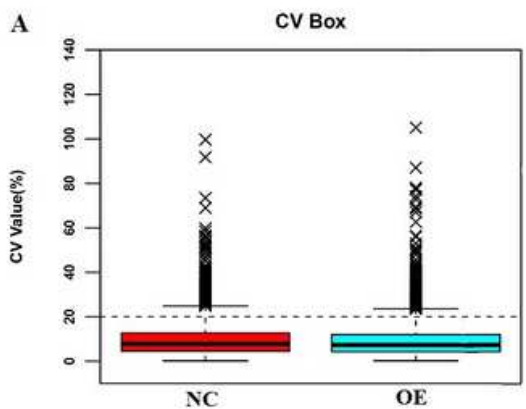

C
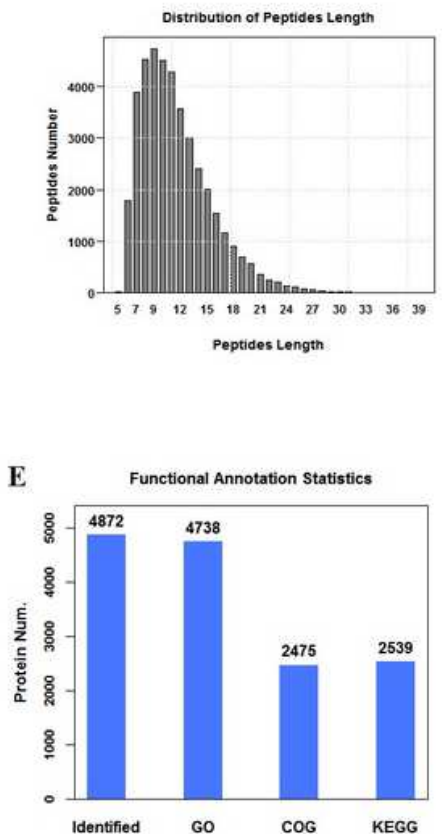

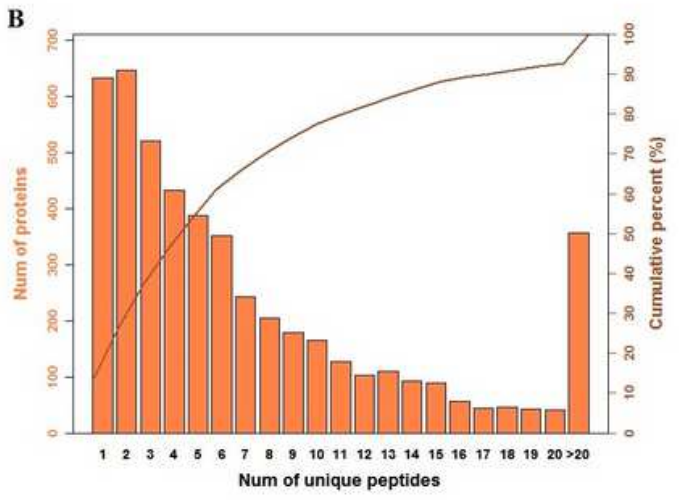

D

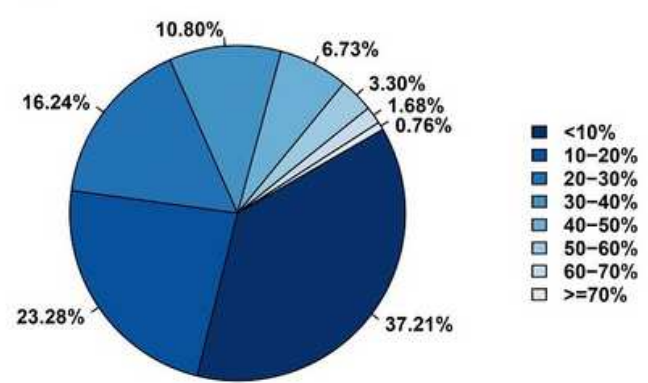

$\mathbf{F}$

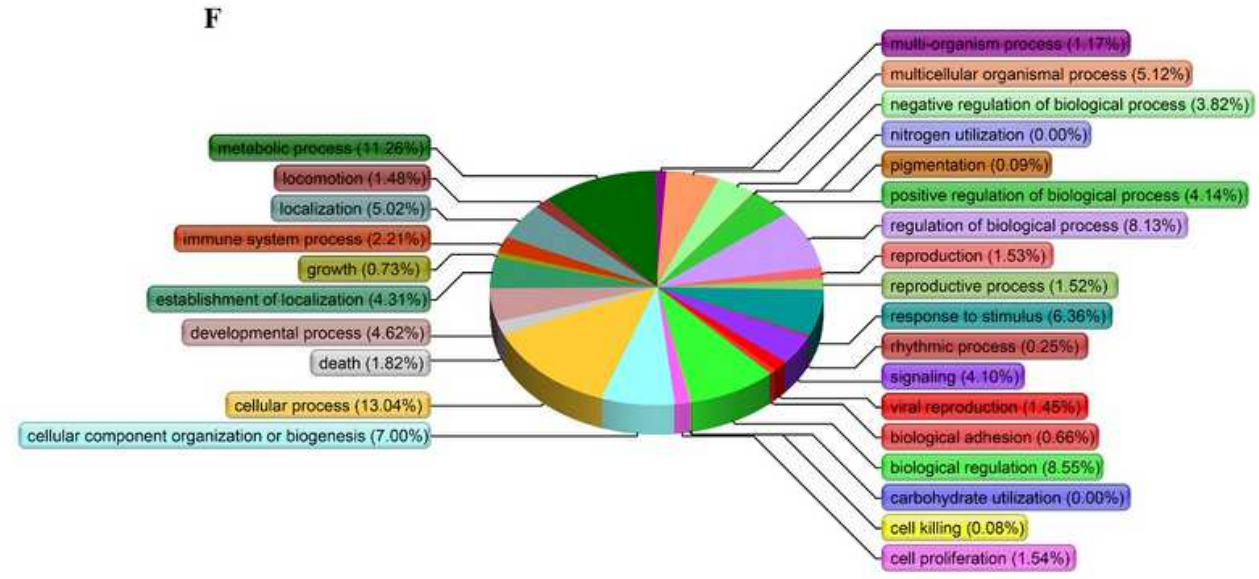

\section{Figure 3}

The summary of the iTRAQ-based proteomic analysis for HIF-AS1 overexpression (OE) and normal control (NC) groups. (A) A shows the comparison of the CV values of different experimental groups more intuitively through the $\mathrm{CV}$ box diagram. The box diagrams of different colors represent the $\mathrm{CV}$ distribution of different experimental groups. The median CV of each box from left to right is $7.81 \%$ and $7.29 \%$, respectively. (B) Distribution of the number of unique peptides obtained for all the proteins identified in this assay. (C) Peptide length distribution map of the identified peptides. (D) Sectors of different colors in the $p$ ie chart representing the percentage of protein with different ranges of identified coverage coverage. (E) Statistical graph of different functions annotation results of. The abscissa indicates identification or different annotation methods, and the ordinate indica tes the number of proteins corresponding to the abscissa. (F) GO analysis of the proportion of proteins involved in various biological processes. 
A

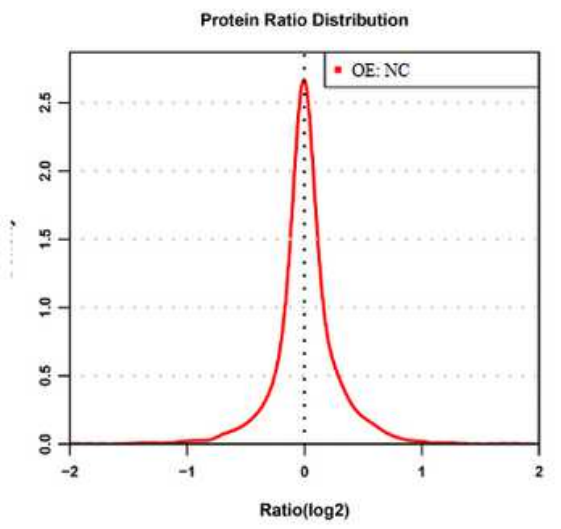

B

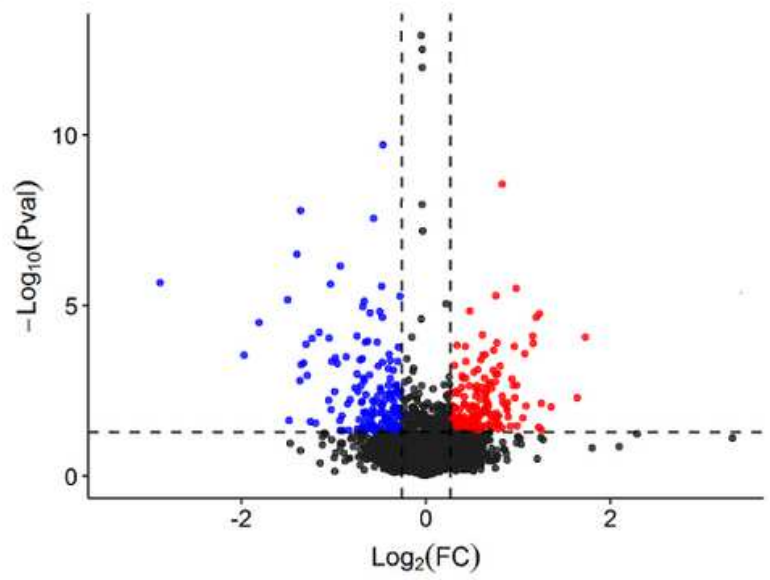

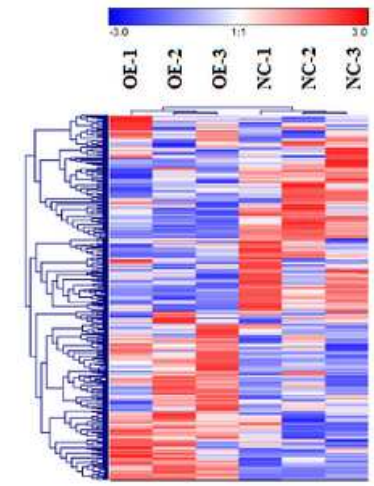

D

Statistics of Up-Regulated Proteins

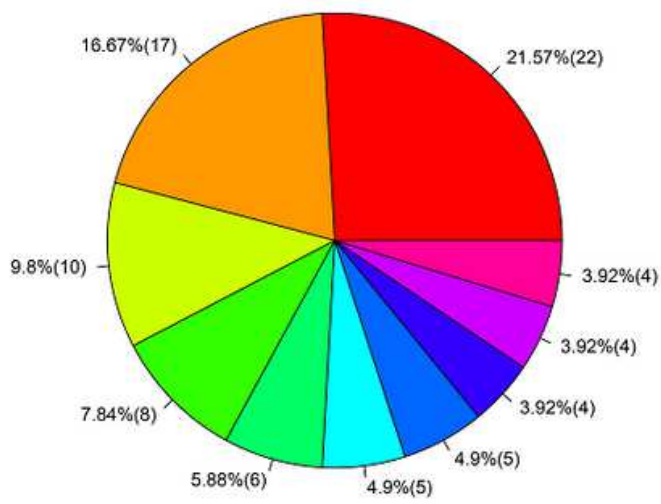

- RNA transport

Metabolic pathways

Ribosome

Spliceosome

Microbial metabolism in diverse environments

- Pathogenic Escherichia coli infection

- Protein processing in endoplasmic reticulum

- Regulation of actin cytoskeleton

- Glycolysis / Gluconeogenesis

- Focal adhesion
Statistics of Down-Regulated Proteins

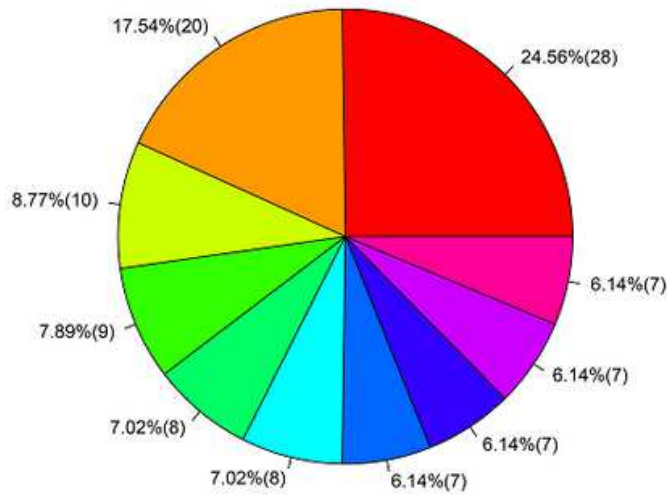

- Metabolic pathways

- Protein processing in endoplasmic reticulum

- Pathways in cancer

- Arrhythmogenic right ventricular cardiomyopathy (ARVC)

- Regulation of actin cytoskeleton

- Microbial metabolism in diverse environments

- Hepatitis C

- Hypertrophic cardiomyopathy (HCM)

- Tight junction

- Dilated cardiomyopathy

\section{Figure 4}

Exploration of differentially expressed proteins (DEPs) and functional analysis. (A) Analyzing distribution of quantifiable multiples about difference proteins, the abscissa represents the value of multiples of difference after logarithmic transformation with base 2 as the base. The expression level greater than 0 shows up-regulated, and the expression level less than 0 shows down-regulated. (B) Volcano plot showing the distribution of proteins that are up regulated (red) and down regulated (blue) or showed no change (black) on overexpression of HIF AS 1. (C) Hierarchical clustering of prote ins vs samples where rows represent the clustering of proteins, and columns represent the clustering of samples. As the protein abundance ratio changes from small to large, the heat map color shows a corresponding blue white red change. (D) KEGG analysis of the proportion of proteins involved in various biological processes. 


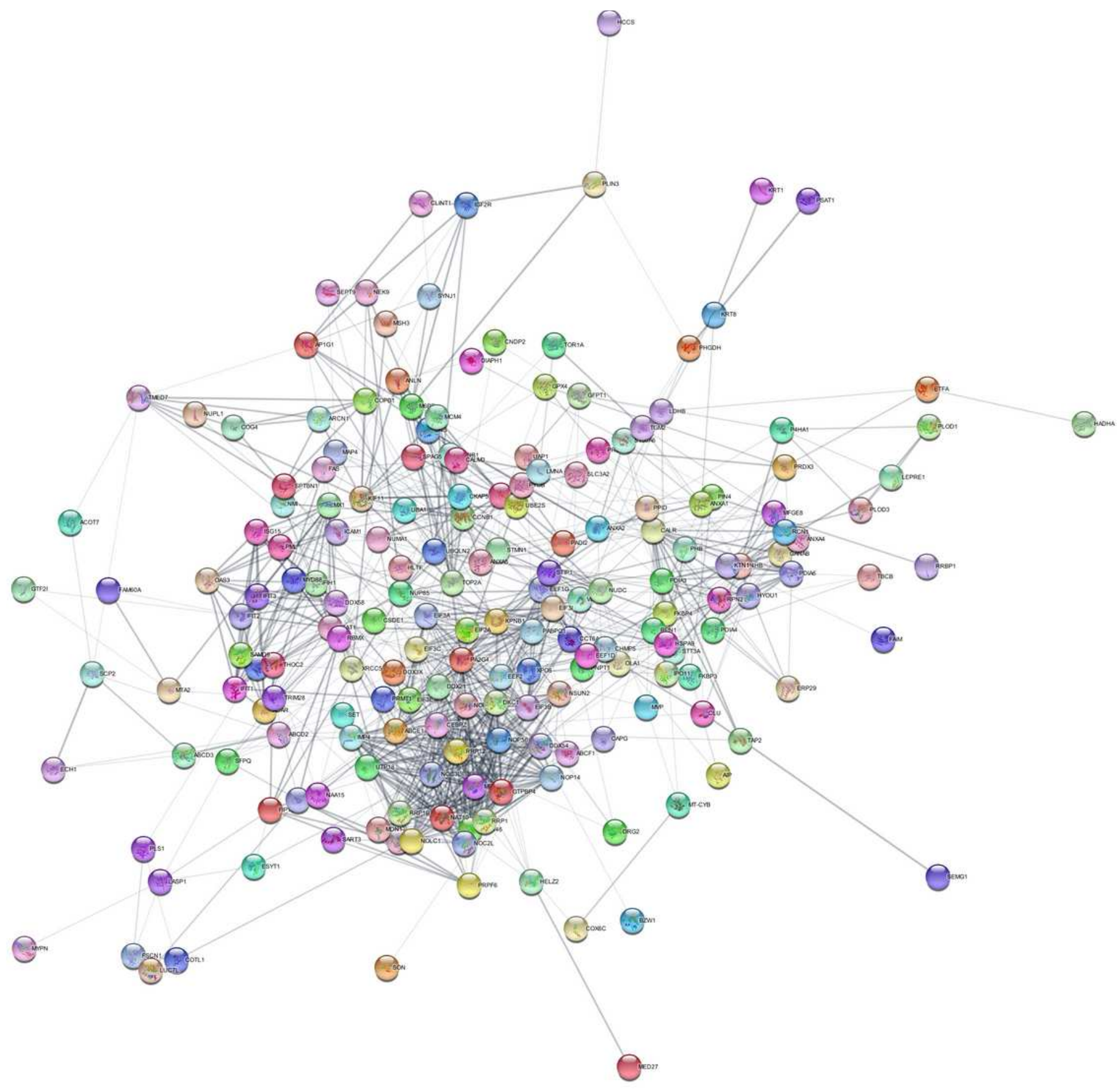

Figure 5

Protein-protein interaction (PPI) network based on the differentially expressed proteins (DEPs). The total of 338 DEPs were filtered into the DEPs PPI network complex using the STRING online database. The round nodes indicate individual proteins. 
A

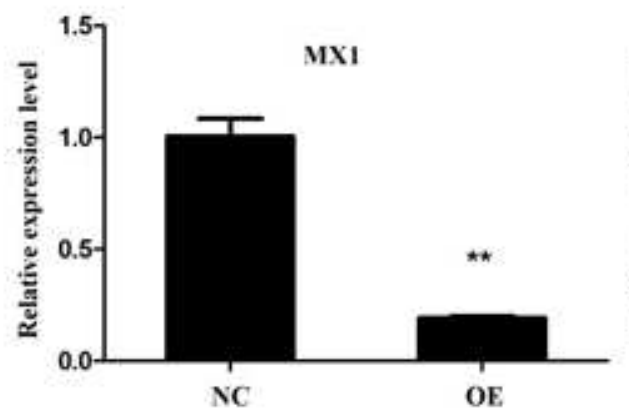

D

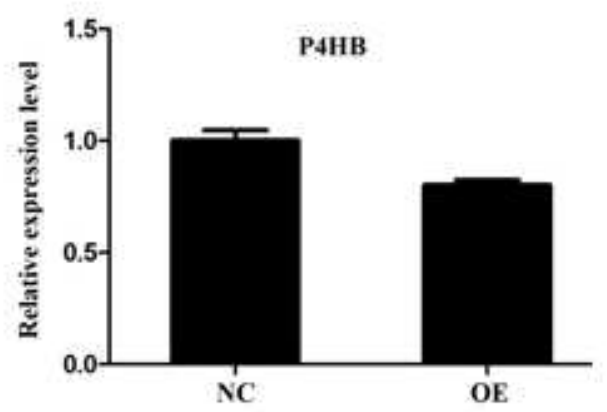

B

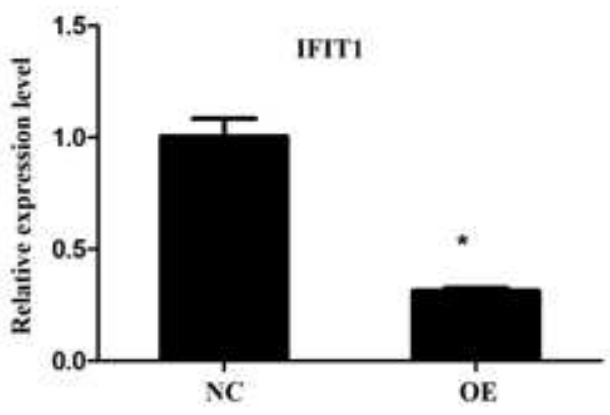

$\mathrm{E}$

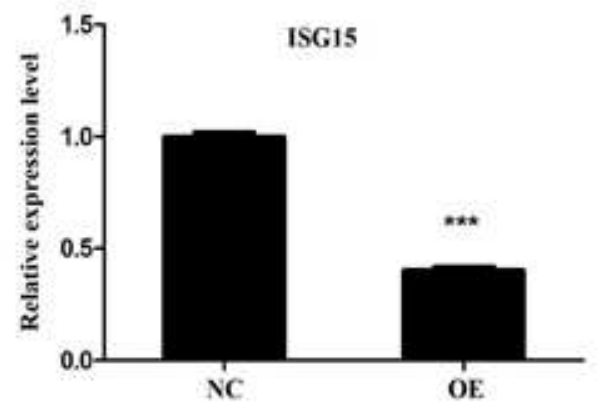

$\mathrm{C}$

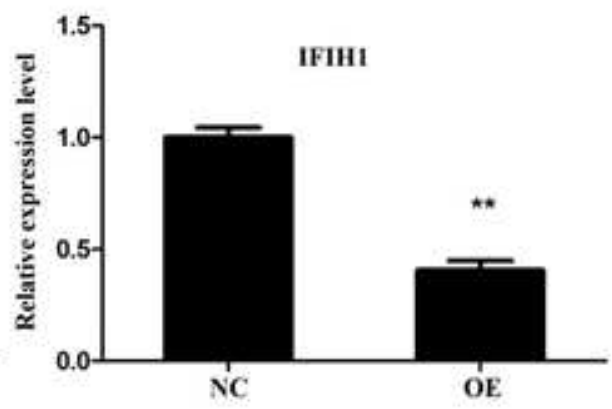

$\mathrm{F}$

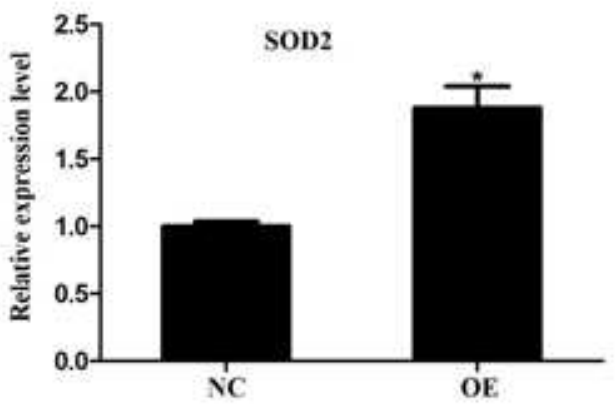

\section{Figure 6}

The quantitative RT-PCR (qRT-PCR) validation of some differentially expressed proteins (DEPs) obtained from iTRAQ analysis in Overexpression (OE) of HIF-AS1 and normal control (NC) groups. The expression levels of the same DEGs including MX1 IFIH1 IFIT1 P4HB ISG15 and SOD2 were validated by RT $\triangle$ qPCR and normalized against Actin expression level. Values are normalized to those from cells with control vector. $n=3$, paired student's $t$ test, $\pm S D$. ${ }^{\star} P<0.1,{ }^{*} P<0.05$, ${ }^{\star *} * P<M X 1$, Interferon induced GTP binding protein $\mathrm{Mx} 1 ; \mathrm{IFIH} 1$, Interferon induced helicase $\mathrm{C}$ domain containing protein 1; IFIT1, Interferon induced protein with tetratricopeptid e repeats 1; ISG15, Ubiquitin like protein ISG15; P4HB, Protein disulfide isomerase; SOD2, Superoxide dismutase [Mn],mitochondrial.

\section{Supplementary Files}

This is a list of supplementary files associated with this preprint. Click to download.

- Table2.xlsx

- Table3.xlsx

- Table4.xlsx

- supplementary.xlsx 